\title{
Influence of Interfacial Oxidation on Friction in Structural Superlubricity
}

\author{
Alper Özoğul ${ }^{1} \cdot$ Benedykt R. Jany ${ }^{2} \cdot$ Franciszek Krok $^{2} \cdot$ Enrico Gnecco $^{1} \cdot$ Mehmet Z. Baykara $^{3}$ (i)
}

Received: 16 March 2021 / Accepted: 22 June 2021 / Published online: 16 July 2021

(c) The Author(s) 2021

\begin{abstract}
We present the results of friction experiments performed by manipulation of oxidized platinum nanoislands on highly oriented pyrolytic graphite (HOPG) substrates through atomic force microscopy (AFM). The oxidation of the platinum nanoislands, performed via mild plasma exposure, is confirmed through X-ray photoelectron spectroscopy (XPS) and high-resolution energy-dispersive X-ray spectroscopy (EDX), the latter of which reveals partial oxidation on the sliding surfaces of the nanoislands. Oxidized platinum nanoislands are found to exhibit higher friction than non-oxidized islands, with a 70\% increase in mean shear stress over the investigated contact size regime. An increase in chemical interaction forces between the oxidized platinum and the graphite substrate is proposed to explain the increase in friction forces. Our results reveal that alteration of interfacial chemistry through oxidation leads to a noticeable modulation of friction forces, but not a total breakdown of the superlubric state (as evidenced by the signature observation of decreasing shear stress with increasing contact size), providing further feasibility for the design of superlubric mechanical systems to be operated under ambient conditions.
\end{abstract}

\section{Graphic Abstract}
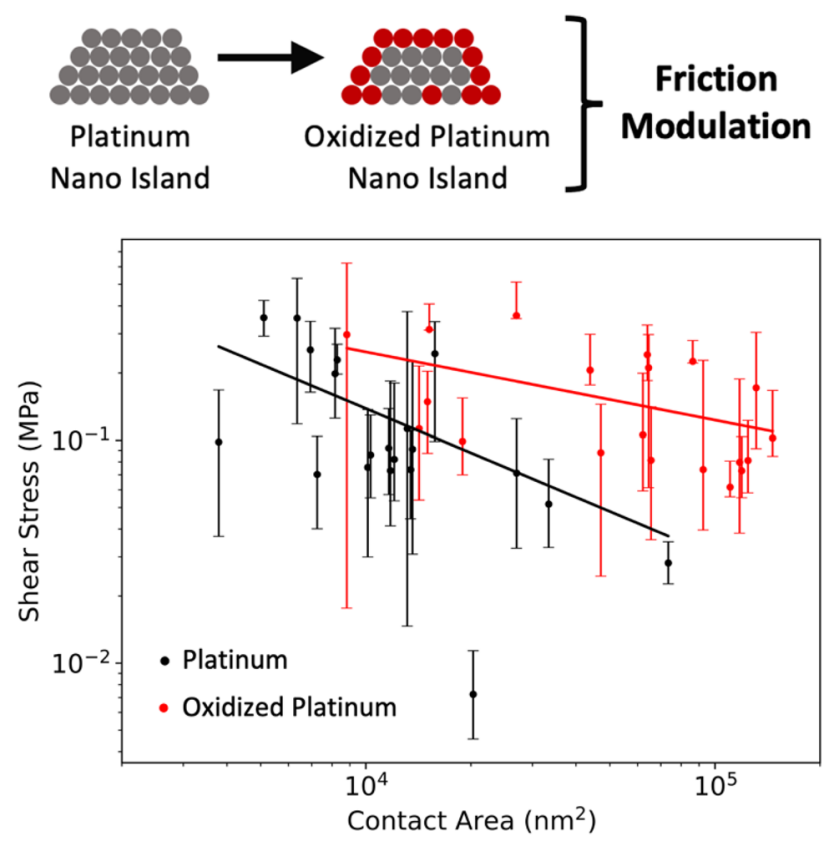

Keywords Atomic force microscopy $\cdot$ Electron microscopy $\cdot$ Friction $\cdot$ Nanotribology $\cdot$ Oxidation $\cdot$ Superlubricity

Mehmet Z. Baykara

mehmet.baykara@ucmerced.edu

Extended author information available on the last page of the article 


\section{Introduction}

A particularly important grand challenge for humanity in the twenty-first century will be to drastically reduce our consumption of fossil fuel sources for energy. While research in renewable energy sources made great strides in the past few decades and are playing a major role toward this arduous task [1], a complementary approach involves searching for improvements in energy efficiency, e.g., by striving to minimize energy dissipation in mechanical systems through friction. Considering the tremendous amount of useful mechanical energy that is "lost" every day through friction in almost any mechanical system one can think of [2], the significance of devising novel ways to minimize friction quickly becomes apparent.

Despite the importance of minimizing friction from economic and environmental points of view, our lack of a comprehensive understanding of the phenomenon prevents the formation of universal strategies that can be applied toward this goal. As interfaces formed between components on the conventional engineering scale are complex, mainly due to the fact that they consist of multiple-asperity contacts, the number, size and mechanical properties of which are not known a priori, fundamental friction research aiming to identify physical mechanisms is almost always performed on much smaller length scales (from the micro- to the nanoscopic regime), where a certain degree of control over contact geometries can be established [3].

Atomic force microscopy (AFM) experiments in which nanoscale objects (e.g., nanotubes, nanoparticles, and pancake-like nanoislands) are manipulated (i.e., laterally moved) over certain substrates play an important role in fundamental friction research $[4,5]$. Such experiments, in contrast to conventional AFM-based friction measurements where contact geometries/chemistries at the tipsample junction remain largely uncharacterized [6], allow researchers to carefully study friction on the nanometer scale as a function of multiple parameters including but not limited to contact size, shape, and chemistry. It was in fact through such experiments that it was first discovered that gold and antimony nanoislands slide in superlubric fashion (i.e., with ultralow friction forces) on highly oriented pyrolytic graphite (HOPG) substrates under ultrahigh vacuum (UHV) conditions [7, 8]. These measurements confirmed earlier theoretical predictions that atomically flat but structurally incommensurate interfaces free of contaminants should slide with negligible friction forces, in a regime that was named as structural (super) lubricity $[9,10]$. Despite the success of this work, not too much of a connection was formed to realistic applications, mainly due to the perceived limitation to molecularly clean conditions that could not be established under ambient conditions $[11,12]$. As such, the more recent discovery that gold and platinum nanoislands (prepared via thermal evaporation of thin films under vacuum followed by annealing) exhibited structural superlubricity on HOPG under ambient conditions, came as a surprise to the scientific community $[13,14]$. The striking observation of structural superlubricity under ambient conditions for these material systems was attributed to the formation of atomically flat and crystalline sliding interfaces that were predicted, via ab initio calculations, to resist contamination by ambient molecules [13]. Despite the fact that both gold and platinum nanoislands exhibited structural superlubricity on HOPG, a significant difference was observed in friction forces: platinum nanoislands experienced roughly four times the friction force experienced by gold islands of comparable size while sliding on HOPG. Ab initio calculations pointed toward the occurrence of stronger chemical interactions between platinum and HOPG, when compared with gold and HOPG, as the likely reason for the observation of increased friction forces [14]. This result underlined the important influence of interfacial chemistry on friction forces in the structural superlubricity regime.

Within this context, an important but so far unanswered question is the following: Can we tune friction forces in the structural superlubricity regime by modulating interfacial chemistry? Equally importantly, as we know that surfaces of most metals chemically degrade under environmental conditions with time, do we expect a common phenomenon such as oxidation to affect, or perhaps lead to a complete breakdown of superlubric sliding? To take preliminary steps toward the answering of these important questions, we present here AFM-based friction experiments performed on platinum nanoislands on HOPG, whereby the surfaces of the islands have been oxidized through mild plasma exposure. Specifically, we discover that partial oxidation at the slider-substrate leads to a noticeable increase in friction force and shear stress, but not a total breakdown of the superlubric, ultralow friction state.

\section{Materials and Methods}

Platinum nanoislands were grown on freshly cleaved, commercially available ZYB-grade HOPG substrates via e-beam evaporation of a thin $(\sim 1 \mathrm{~nm})$ film under high vacuum, followed by post-deposition annealing for $30 \mathrm{~min}$ at $1000{ }^{\circ} \mathrm{C}$ as described before [14]. An alternative growth method was also tested, where another batch of platinum nanoislands, with a nominal coverage of $1 \mathrm{~nm} \mathrm{Pt}$, were synthesized by magnetron sputtering deposition on HOPG which was kept at $564{ }^{\circ} \mathrm{C}$ during deposition, similar to a method described earlier [15]. Comparison of the morphology and structure of the platinum nanoislands grown by these two methods 
yielded no noticeable differences as checked by Scanning Electron Microscopy (SEM) imaging and Electron Backscatter Diffraction (EBSD), performed using a Dual Beam SEM/FIB microscope equipped with the EDAX/Ametek EDX/EBSD analysis system (Quanta 3D FEG, FEI). The EBSD data were collected at $20 \mathrm{keV}$ electron energy; only the data with a confidence index (CI) of $>0.1$ were selected for further analysis. To oxidize the nanoislands, samples prepared in the fashion described above were exposed to oxygen plasma in a reactive ion etching (RIE) system, under $50 \mu \mathrm{B}$ ar of plasma pressure and at $50 \mathrm{~W}$ power for a duration of $2 \mathrm{~min}$. The oxidation of the platinum nanoisland surfaces was confirmed via X-Ray photoelectron spectroscopy (XPS) and additionally through high-resolution, cross-sectional energy-dispersive X-ray spectroscopy (EDX) mapping performed in a scanning transmission electron microscope (STEM) operated at $200 \mathrm{keV}$ ((S)TEM Titan3 G2 60-300, FEI). The STEM imaging was performed in the High Angle Annular Dark Field (HAADF) mode where the signal intensity is proportional to atomic number and to sample thickness. XPS spectra were calibrated by measuring the binding energy of the $\mathrm{C} 1 \mathrm{~s}$ orbital, and comparing it with reference values from the literature $[16,17]$. The samples for STEM measurements were prepared using Focused Ion Beam (FIB). Friction experiments were performed via lateral manipulation of the nanoislands on the HOPG surface in contact-mode AFM (XE-100E, PSIA); the details of the related procedure were previously described $[13,18]$. In essence, the AFM tip scanning over the surface occasionally makes contact with a nanoisland, pushing it from the side and thus moving (i.e., manipulating) it over the HOPG substrate. As soon as the manipulation starts, an increase in the lateral force signal experienced by the AFM cantilever is recorded, which manifests as a bright "streak" in the lateral force map and directly corresponds to the friction force acting at the nanoisland-HOPG interface. The mean and standard deviation values of friction associated with each island are extracted from these lateral force profiles. In line with previous work, contact areas between the nanoislands and the HOPG substrate were determined simply from the AFM-based topographical imaging of the nanoislands, whereby error bars of $\pm 10 \%$ have been included to account for potential tip shape effects [19]. The lateral calibration of the cantilevers employed in the AFM experiments (ContAl$\mathrm{G}$, BudgetSensors) was performed via the Ogletree method [20]. No normal force was applied on the cantilevers during the measurements, i.e., the net normal force consisted of adhesion only.

\section{Results and Discussion}

To directly probe the influence of interfacial chemistry on friction in the structural superlubricity regime, and motivated by previous studies of platinum oxidation in the literature $[16,17]$, we oxidized the surfaces of our platinum
Fig. 1 Structural characterization of oxidized platinum nanoislands via electron microscopy. a SEM image of oxidized platinum nanoislands on HOPG. b High-resolution, cross-sectional HAADF STEM image of an oxidized platinum nanoisland showing pristine atomic planes in the bulk, and the corresponding diffraction pattern (inset). Note that the presence of any oxide layers on the nanoisland surfaces cannot be clearly detected in the TEM images. c SEM EBSD Inverse Pole Figure (IPF) ( $z$ out of plane) showing that all platinum nanoislands grow by exposing their (111) planes in parallel fashion toward the HOPG surface. Scale bars correspond to $200 \mathrm{~nm}, 3 \mathrm{~nm}$, and $600 \mathrm{~nm}$, respectively
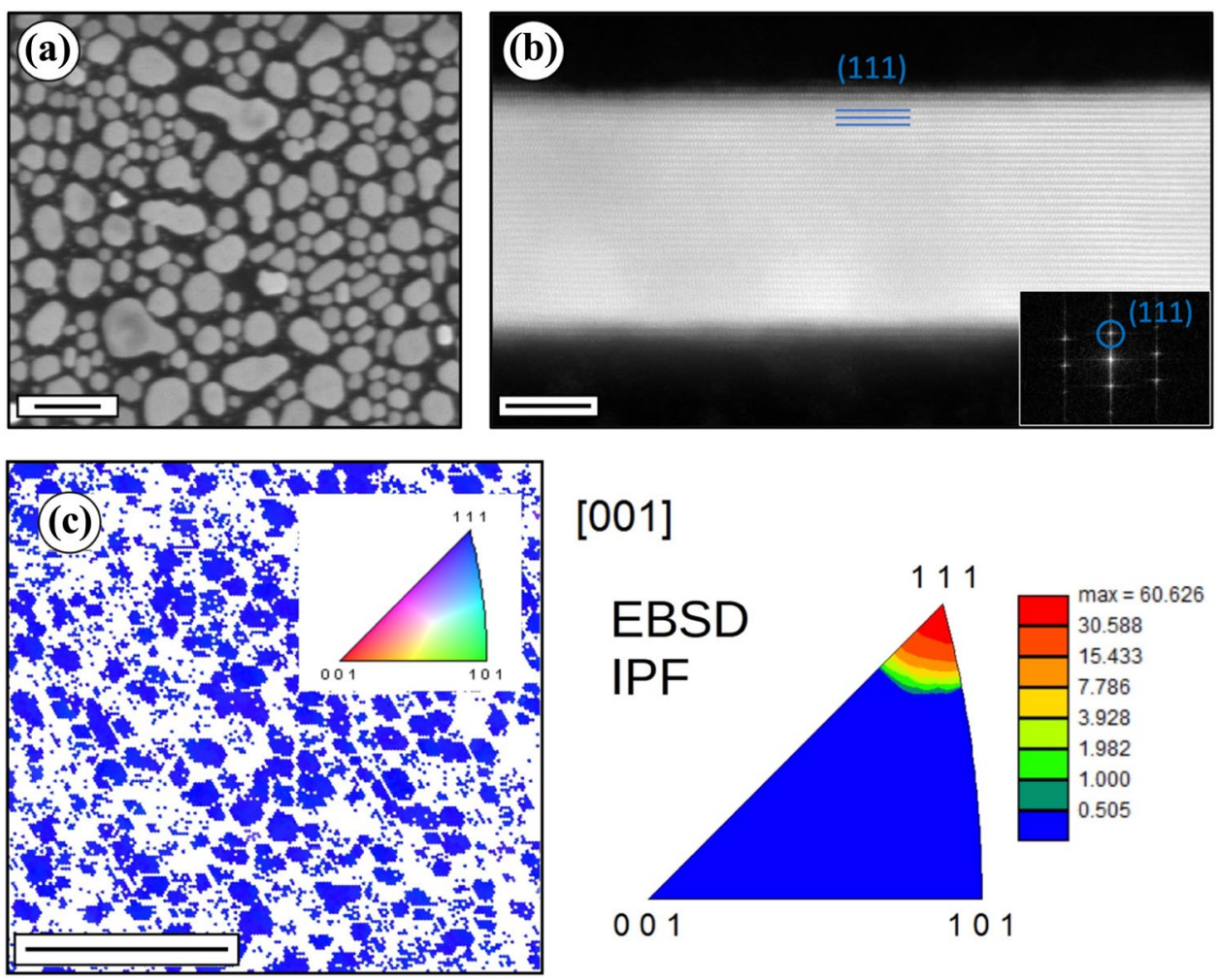

[001]

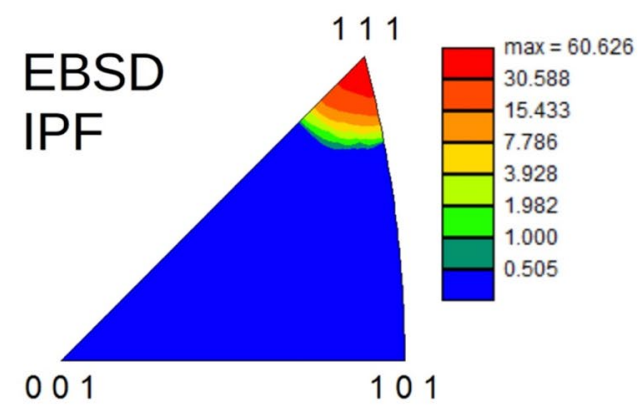


nanoislands as described in Sect. 2. Figure 1a presents a representative scanning electron microscopy (SEM) image of the HOPG substrate decorated with oxidized platinum nanoislands. By way of such SEM images, it can be confirmed that the oxidized platinum nanoislands preserve the flat, "pancake-like" morphology of their parent platinum particles (attributed to the relatively short durations of plasma exposure) [14], spanning a lateral size regime from a few tens of $\mathrm{nm}$ to a few hundreds of $\mathrm{nm}$. It should be additionally noted that while the HOPG surface appears to be densely packed with nanoislands, sufficiently wide, bare areas can be found that allow adequate lateral manipulation of islands for friction measurements. Finally, while the oxidized nanoislands often expose straight edges, indicative of crystallinity, we additionally performed SEM EBSD measurements, as well as high-resolution, cross-sectional HAADF STEM imaging on several nanoislands to confirm their structure. A representative HAADF STEM image, presented in Fig. 1b, clearly shows atomic planes arranged on top of each other in (111) configuration, parallel to the top surface of the HOPG substrate. The corresponding Fourier Transform (Fig. 1b, inset) provides further validation for the crystalline structure of the nanoisland. Additionally, the SEM EBSD Inverse Pole Figure (IPF) ( $z$ out of plane) in Fig. 1c clearly shows that all nanoislands are in (111) configuration with respect to the HOPG surface.

The fact that the cross-sectional HAADF STEM images show predominantly pristine (111) planes in the nanoislands leads to the conclusion that the oxidation must have been limited to a very thin region on the island surfaces; as such, the method of XPS was utilized to confirm the surface oxidation of the nanoislands. Toward this purpose, XPS spectra were obtained for $\mathrm{Pt} 4 \mathrm{f}, \mathrm{C} 1 \mathrm{~s}$, and $\mathrm{O} 1 \mathrm{~s}$ regions in the plasma-treated samples, whereby a representative spectrum in the Pt4f region is provided in Fig. 2. Here, metallic Pt4f doublet peaks (blue) are accompanied by a further doublet peak (red), which corresponds to $\mathrm{PtO}_{(7 / 2)}$ and confirms that our platinum nanoislands are indeed oxidized $[16,17]$. Moreover, no significant change in the $\mathrm{C} 1 \mathrm{~s}$ spectrum was observed after oxidation (please see Fig. S1), suggesting that the chemical characteristics of the HOPG substrate remained unchanged after the brief exposure to oxygen plasma.

Despite the fact that the XPS results presented in Fig. 2 clearly indicate oxidation, the technique is limited by penetration depths of a few $\mathrm{nm}$ on the platinum nanoislands, and as such the related results only confirm that the top surfaces of the islands are oxidized and do not provide useful information about the bottom surfaces of the islands that form the sliding interface with the HOPG substrate. As friction on the nanoscale is mainly dominated by the structural and chemical characteristics of the slider and substrate surfaces [21], a more careful investigation of oxidation at the bottom surfaces of islands is of crucial importance. Motivated in this

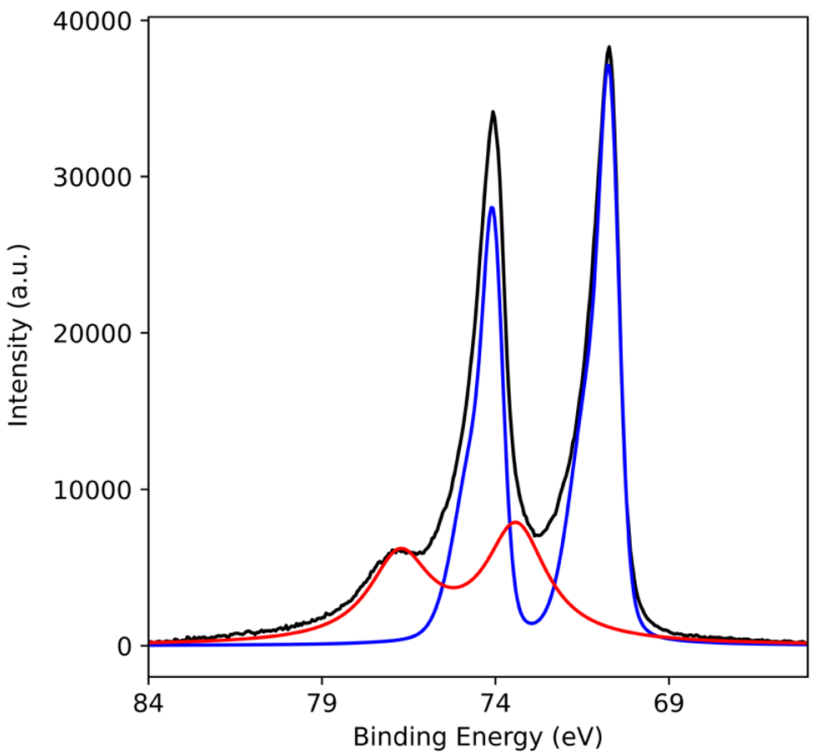

Fig. 2 XPS investigation of the oxidized platinum nanoislands. The experimentally measured spectrum (background subtracted) is shown in black, whereas the reference spectrum corresponding to the metallic Pt4f orbital is shown in blue, and the reference spectrum corresponding to $\mathrm{PtO}_{(7 / 2)}$ is shown in red (Color figure online)

fashion, we acquired high-resolution EDX maps on oxidized platinum nanoislands that were prepared via FIB and investigated in a cross-sectional STEM configuration. A representative result is shown in Fig. 3, where the cross-sectional HAADF STEM image belonging to a particular nanoisland is presented together with the corresponding EDX map of oxygen and a line profile showing oxygen intensity along the cross-section of the island. As one can see in Fig. 3, a clear oxygen signal is detected both on the top and the bottom (i.e., sliding) surfaces of the nanoisland. At this point, a couple of observations need to be highlighted: (i) the overall intensity of oxygen is typically higher on the top surface than the bottom surface, (ii) the intensity of oxygen on the bottom surface changes depending on lateral position, and (iii) while the majority of islands we investigated showed recognizable oxygen content on the bottom surfaces, there were some islands where oxidation of the bottom surface could not be clearly recorded. Point (i) can be quickly rationalized when one considers that the top surfaces of the islands are fully exposed during the plasma oxidation process while the bottom surfaces are (partially) protected by the HOPG substrate. On the other hand, while point (ii) indicates a nonuniform oxidation of the bottom surface, point (iii) can be understood when one takes into account the variability in the way that the FIB-based cross-sectioning procedure is performed. Specifically, considering that the oxygen plasma penetrates the island-substrate interface at island edges, FIB cross sections performed closer to the edges of a particular island would naturally show a higher degree of oxidation at 
Fig. 3 a Cross-sectional HAADF STEM image of an oxidized platinum nanoparticle. b The corresponding EDX map for the oxygen $\mathrm{K} \alpha$ peak, where brightness indicates a higher oxygen count (background subtracted). Scale bar corresponds to $15 \mathrm{~nm}$. c Line profile of oxygen intensity taken along the white, dashed arrow in (a), from the top to the bottom of the nanoparticle. Two peaks corresponding to increased oxygen content on the top $(\sim 5000$ counts) and the bottom surfaces ( 2500 counts) can be observed (Color figure online)
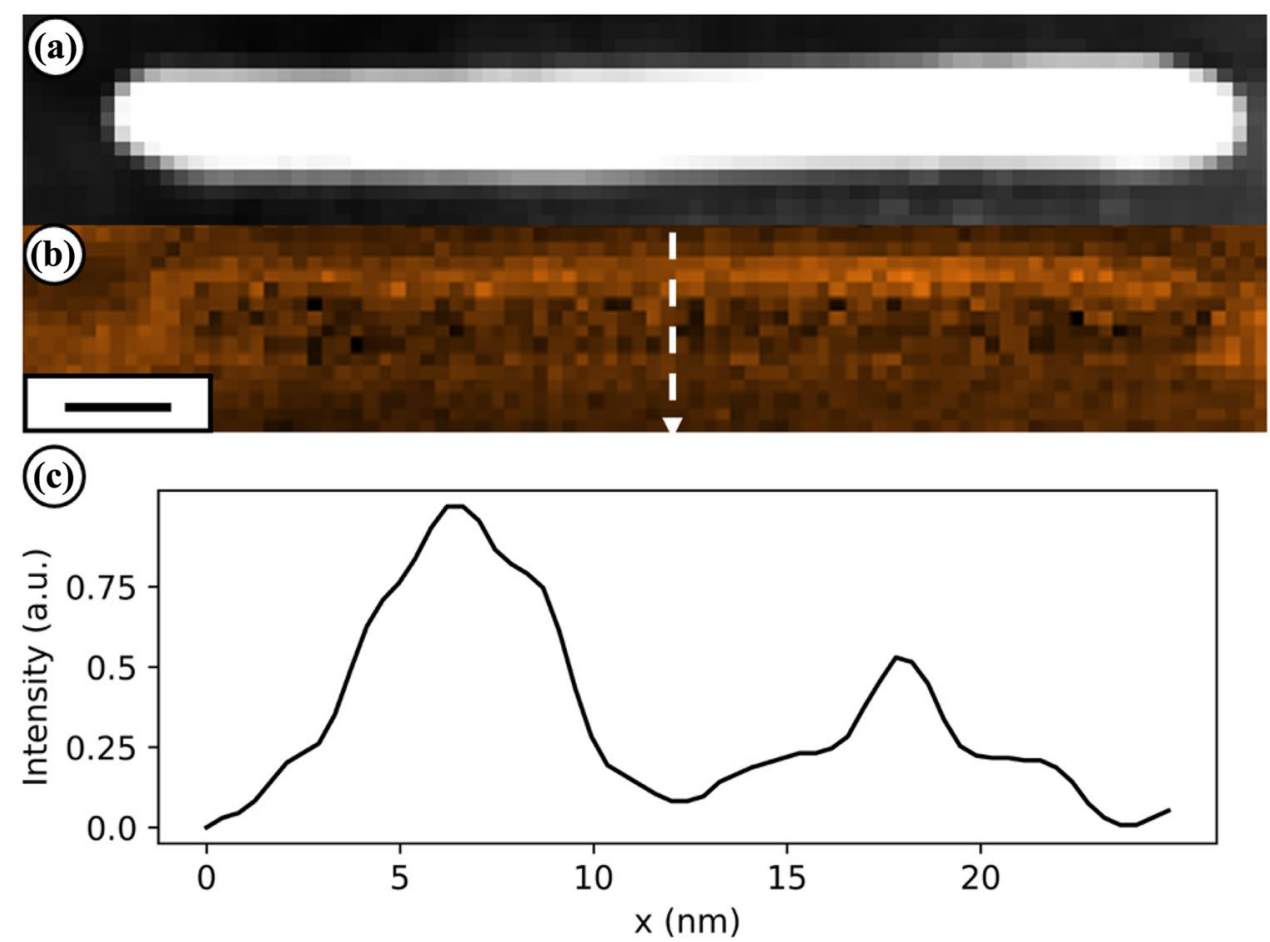

Table 1 RMS roughness values for the HOPG substrate pre- and post-oxidation, as well as the non-oxidized and oxidized platinum nanoislands

\begin{tabular}{lc}
\hline & RMS roughness (nm) \\
\hline HOPG, pre-oxidation & $0.5 \pm 0.2$ \\
HOPG, post-oxidation & $0.5 \pm 0.2$ \\
Platinum nanoislands & $5.5 \pm 0.4$ \\
Oxidized platinum nanoislands & $13.6 \pm 1.4$ \\
\hline
\end{tabular}

the bottom surface, whereas slices performed closer to the middle region of the islands may not show too much oxidation at all. Regardless, our results demonstrate that oxygen ions can indeed penetrate the island-substrate interface and lead to a chemical alteration of the sliding interface.

As the final step in the characterization of the sample system, we performed AFM-based topographical scans (on both the HOPG substrate and the nanoisland surfaces) preand post-oxidation, from which root-mean-square (RMS) roughness values were extracted (see Table 1). Although no change in the RMS roughness of the HOPG substrate is detected after the brief plasma exposure $(0.5 \pm 0.2 \mathrm{~nm})$, the roughness of the top surface of the nanoislands increases significantly (from $5.5 \pm 0.4 \mathrm{~nm}$ to $13.6 \pm 1.4 \mathrm{~nm}$ ). It should be noted that the RMS roughness measured on the islands are often affected by bump-like topographical features which form during the growth process, hence the relatively high values when compared with the atomically flat HOPG.
Friction experiments were then conducted on the nanoislands, whereby the friction force experienced by each nanoisland was measured as it was manipulated on the HOPG substrate by the tip of the AFM, together with the corresponding contact area, as described in Sect. 2. Results are reported in Fig. 4a, together with results obtained on gold and non-oxidized platinum nanoislands presented in previous work [13, 14]. A particular observation can be readily made by inspecting the data presented in Fig. 4a: on average, oxidized platinum nanoislands experience higher friction forces than platinum and gold nanoislands. In particular a mean friction of $9.25 \mathrm{nN}$ is measured for oxidized platinum nanoislands, whereas mean friction values of $1.41 \mathrm{nN}$ and $0.33 \mathrm{nN}$ are reported for platinum and gold nanoislands, respectively. On the other hand, it needs to be taken into account that a significant number of oxidized platinum nanoislands investigated here are larger than the majority of platinum and gold nanoislands, as underlined by the fact that they are accumulated on the right side of the plot. Considering that friction scales with contact area in the structural superlubricity regime [22], a more rational comparison between the friction values experienced by the three types of islands can be made by dividing Fig. $4 \mathrm{a}$ into two regions. In particular, Region 1 contains oxidized platinum nanoislands that are of comparable size to the great majority of gold and platinum nanoislands, whereas Region 2 contains oxidized platinum nanoislands that are significantly larger. Once this is done, it is observed that oxidized platinum 

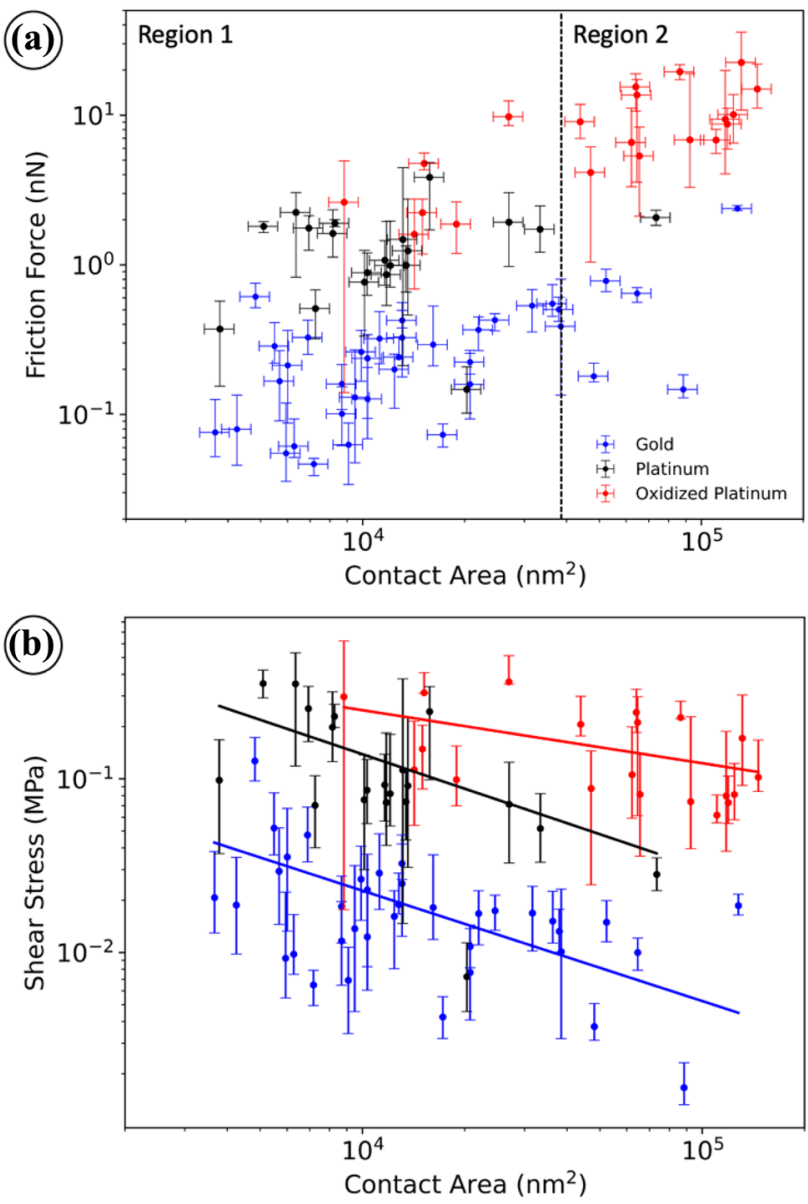

Fig. 4 a Friction force measured as a function of interfacial contact area for gold (blue), platinum (black) and oxidized platinum (red) nanoislands. The dashed line separates Regions 1 and 2, as described in the text. b Shear stress measured as a function of interfacial contact area for gold (blue), platinum (black) and oxidized platinum (red) nanoislands. Lines are best fits to the data that represent scaling of shear stress with respect to contact area according to the relation $\tau=c_{1} A^{c_{2}}$ (Color figure online)

Table 2 Friction forces and shear stresses experienced by the three types of nanoislands. For oxidized platinum nanoislands, the reported friction force corresponds to islands in Region 1 of Fig. 4a

\begin{tabular}{lll}
\hline & Friction force $(\mathrm{nN})$ & Shear stress $(\mathrm{MPa})$ \\
\hline Gold [13] & $0.33 \pm 0.39$ & $0.02 \pm 0.02$ \\
Platinum [14] & $1.41 \pm 0.83$ & $0.13 \pm 0.10$ \\
Oxidized platinum & $4.14 \pm 2.77$ & $0.22 \pm 0.30$ \\
\hline
\end{tabular}

nanoislands in Region 2 experience a mean friction force of $11.8 \mathrm{nN}$, while those in Region 1 experience a mean friction force of $4.14 \mathrm{nN}$. Compared with the mean friction force reported for platinum nanoislands (please see Table 2 for a summary of mean and standard deviation values of friction forces for all types of nanoislands discussed here), we arrive at the conclusion that interfacial oxidation leads to a noticeable increase in friction for platinum nanoislands.

While ultralow friction forces are an indication of superlubricity, it needs to be mentioned that the magnitude of the friction force is a function of both contact area and the strength of chemical interaction between the slider and the substrate in the structural superlubricity regime. As such, a direct comparison of friction forces experienced by nanoislands composed of different materials should not yield a straightforward conclusion about the persistence or breakdown of structural superlubricity. Instead, shear stresses (i.e., friction force per contact area) need to be inspected for a clear proof of structural superlubricity. Shear stresses also provide a better means of comparing islands of different size in terms of frictional behavior than friction forces. To follow up from the first point, let us recall that in the structural superlubricity regime, friction forces scale sub-linearly with contact size; in other words, shear stress is expected to decrease with increasing contact area [22, 23]. To check whether this theoretical prediction is valid for our data, we calculated shear stresses $(\tau)$ for the gold, platinum, and oxidized platinum nanoislands discussed here and plotted them as a function of contact area $(A)$ in Fig. 4b, along with best fits in the form of $\tau=c_{1} A^{c_{2}}$. The results clearly show a decreasing trend for all three types of nanoislands and thus validate that the ultralow friction forces we recorded are indeed in agreement with the theory of structural superlubricity. On the other hand, it can be seen that although the shear stress for the oxidized platinum islands is decreasing with contact area, this occurs with a significantly weaker slope, i.e., with a scaling factor $c_{2}(-0.31 \pm 0.12)$ that is significantly lower than what has been recorded for gold and platinum islands $(-0.64 \pm 0.25$ and $-0.66 \pm 0.27$, respectively).

We also extracted mean values of shear stress for the three types of islands in our experiments. The results, reported in Table 2, point to a $70 \%$ increase in mean shear stress when platinum nanoislands are oxidized. It should be noted that (i) friction forces recorded by way of experiments described here are susceptible to significant fluctuations (which can be attributed to spontaneous rotations of islands as they are being manipulated by the AFM tip [24]) and (ii) friction in the structurally superlubric regime is not only a function of contact size but also of its shape [22]; hence the rather substantial error bars in the reported friction force and shear stress values.

Motivated by the results described above, the natural next step in our investigation is to determine potential reasons why interfacial oxidation leads to a noticeable increase in shear stresses, and consequently friction forces, experienced by platinum nanoislands. Two main avenues through which friction can increase in the structural 
superlubricity regime are (i) an increase in the magnitude of chemical interaction forces between the slider and the substrate (as was the case when platinum nanoislands were manipulated on HOPG instead of gold nanoislands [14], or when the substrate was switched from HOPG to $\mathrm{MoS}_{2}$ for manipulation experiments on antimony nanoislands [23]) or, (ii) an increase in the topographical roughness of the interface, which naturally involves the evolution of a multiple-asperity contact at the interface from the originally atomically flat, albeit structurally incommensurate geometry. The latter avenue leads to an increase in friction forces, as the pairwise cancellation of lateral forces at a structurally incommensurate interface is not valid for atoms at the edges of the contact: with increasing roughness, we have an increase in the number of asperity contacts, leading to an increase in the number of edge atoms, which in turn leads to an increase in friction [25].

While it is clear from the data presented in Table 1 that the oxidation process leads to a noticeable increase in the roughness of the top surfaces of the nanoislands, it seems unlikely that roughness is the main factor leading to friction increase in our experiments as even a minor increase in roughness at the slider-substrate interface is theoretically expected to eradicate the superlubric state [25], whereas the characteristic negative scaling of shear stress with contact area persists in the oxidized platinum nanoislands. This leaves avenue (i) (i.e., an increase in the magnitude of chemical interaction forces) as the most likely physical reason for the increase in shear stress, and consequently friction forces, measured after oxidation. In fact, it was previously shown in atomistic simulations by $\mathrm{He}$ et al. that oxidation of platinum nanoparticles leads to a drastic ( fivefold) increase in binding energy to graphite surfaces [26]. Considering that the degree of chemical interactions between the slider and the substrate (in addition to contact size, shape and their relative orientation) is one of the main factors that ultimately determine the magnitude of friction forces in structurally superlubric sliding, it is therefore plausible that increased chemical interactions at the slider-substrate interface would lead to enhanced friction forces for oxidized platinum nanoparticles. Having said this, it may come as a surprise that despite the noticeable oxidation at the interface, structural superlubricity still persists, although in a weaker state signified by the strong drop in the shear stress vs. contact area scaling factor when compared with pristine platinum ( -0.31 vs. -0.66$)$. This observation can be tentatively explained by the fact that the bottom, sliding surfaces of the nanoislands appear to be only partially oxidized (Fig. 3), such that the enhanced interactions due to oxidation are valid only for a fraction of the total contact area, leaving open the possibility that a complete breakdown of superlubricity may occur upon full interfacial oxidation.

\section{Conclusions}

Here we presented AFM-based friction experiments performed by manipulation of oxidized platinum nanoislands on HOPG, in order to answer the question of whether changes in interfacial chemistry would lead to a modulation of friction forces in the structural superlubricity regime, or alternatively, to its breakdown. While XPS measurements confirmed oxidation of top surfaces, electron microscopy was utilized to determine that the bottom, sliding surfaces of the islands are partially oxidized, as well. Oxidized platinum nanoislands exhibited a mean shear stress that is $70 \%$ higher than non-oxidized ones when they were manipulated on HOPG. An increase in chemical interaction forces at oxidized regions of the interface was brought forward as the most likely mechanism leading to friction modulation. Our experiments revealed that friction in the structurally superlubric regime can indeed be tuned chemically, e.g., by oxidation, without a total breakdown of the ultralow friction state. The results presented here hint at the possibility of finding material systems where friction could be reversibly modulated in the structurally superlubric regime through chemical means, as well as provide further feasibility for the environmental robustness of superlubric mechanical systems that would be designed to operate under ambient conditions.

Supplementary Information The online version contains supplementary material available at https://doi.org/10.1007/s11249-021-01475-1.

Acknowledgements The authors acknowledge the groundbreaking work of Mark O. Robbins in the fields of nanotribology and superlubricity, and consider it a privilege to publish their work in this collection dedicated to his influential memory. We gratefully acknowledge Prof. A. Kruk from the International Centre of Electron Microscopy for Materials Science, AGH University of Science and Technology in Krakow, for the STEM measurements. We also gratefully acknowledge the help of Dr. Arkadiusz Janas during sample preparation by magnetron sputtering.

Author Contributions MZB, FK, and EG conceived the experiments. AÖ performed the AFM experiments. BRJ performed the SEM/EDX/ EBSD measurements and FIB sample preparation, together with SEM/ EDX/EBSD and STEM/EDX data analysis and interpretation. All authors participated in the final analysis and interpretation of the data.

Funding M.Z.B. acknowledges support by the Marie Curie Actions of the European Commission's FP7 Program in the form of a Career Integration Grant (PCIG12-GA-2012-333843). B.R.J. acknowledges the support of the Polish National Science Centre (NCN) under the grant Miniatura4 DEC-2020/04/X/ST5/00539.

Data Availability The data that support the findings of this study are available from the corresponding author upon request.

\section{Declarations}

Conflict of interest The authors declare that there are no conflicts of interest. 
Open Access This article is licensed under a Creative Commons Attribution 4.0 International License, which permits use, sharing, adaptation, distribution and reproduction in any medium or format, as long as you give appropriate credit to the original author(s) and the source, provide a link to the Creative Commons licence, and indicate if changes were made. The images or other third party material in this article are included in the article's Creative Commons licence, unless indicated otherwise in a credit line to the material. If material is not included in the article's Creative Commons licence and your intended use is not permitted by statutory regulation or exceeds the permitted use, you will need to obtain permission directly from the copyright holder. To view a copy of this licence, visit http://creativecommons.org/licenses/by/4.0/.

\section{References}

1. Panwar, N.L., Kaushik, S.C., Kothari, S.: Role of renewable energy sources in environmental protection: a review. Renew. Sustain. Energy Rev. 15, 1513-1524 (2011)

2. Holmberg, K., Erdemir, A.: Influence of tribology on global energy consumption, costs and emissions. Friction 5, 263-284 (2017)

3. Vanossi, A., Dietzel, D., Schirmeisen, A., Meyer, E., Pawlak, R., Glatzel, T., Kisiel, M., Kawai, S., Manini, N.: Recent highlights in nanoscale and mesoscale friction. Beilstein J. Nanotechnol. 9, 1995-2014 (2018)

4. Schirmeisen, A., Schwarz, U.D.: Measuring the friction of nanoparticles: a new route towards a better understanding of nanoscale friction. ChemPhysChem 10, 2373-2382 (2009)

5. Dietzel, D., Schwarz, U.D., Schirmeisen, A.: Nanotribological studies using nanoparticle manipulation: principles and application to structural lubricity. Friction 2, 114-139 (2014)

6. Szlufarska, I., Chandross, M., Carpick, R.W.: Recent advances in single-asperity nanotribology. J. Phys. D. Appl. Phys. 41, 123001 (2008)

7. Dietzel, D., Ritter, C., Mönninghoff, T., Fuchs, H., Schirmeisen, A., Schwarz, U.D.: Frictional duality observed during nanoparticle sliding. Phys. Rev. Lett. 101, 125505 (2008)

8. Dietzel, D., Feldmann, M., Schwarz, U.D., Fuchs, H., Schirmeisen, A.: Scaling laws of structural lubricity. Phys. Rev. Lett. 111, 235502 (2013)

9. Hirano, M., Shinjo, K.: Atomistic locking and friction. Phys. Rev. B 41, 11837-11851 (1990)

10. Müser, M.H.: Structural lubricity: Role of dimension and symmetry. Europhys. Lett. 66, 97-103 (2004)

11. He, G., Müser, M.H., Robbins, M.O.: Adsorbed layers and the origin of static friction. Science 284, 1650-1652 (1999)

12. Müser, M.H., Wenning, L., Robbins, M.O.: Simple microscopic theory of Amonton's laws for static friction. Phys. Rev. Lett. 86, 1295-1298 (2001)
13. Cihan, E., Ipek, S., Durgun, E., Baykara, M.Z.: Structural lubricity under ambient conditions. Nat. Commun. 7, 1-6 (2016)

14. Özoğul, A., İpek, S., Durgun, E., Baykara, M.Z.: Structural superlubricity of platinum on graphite under ambient conditions: the effects of chemistry and geometry. Appl. Phys. Lett. 111, 211602 (2017)

15. Trillitzsch, F., Guerra, R., Janas, A., Manini, N., Krok, F., Gnecco, E.: Directional and angular locking in the driven motion of $\mathrm{Au}$ islands on $\mathrm{MoS}_{2}$. Phys. Rev. B 98, 165417 (2018)

16. Kim, K.S., Winograd, N., Davis, R.E.: Electron spectroscopy of platinum-oxygen surfaces and application to electrochemical studies. J. Am. Chem. Soc. 93, 6296-6297 (1971)

17. Li, Z., Beck, P., Ohlberg, D.A.A., Stewart, D.R., Williams, R.S.: Surface properties of platinum thin films as a function of plasma treatment conditions. Surf. Sci. 529, 410-418 (2003)

18. Dietzel, D., Mönninghoff, T., Jansen, L., Fuchs, H., Ritter, C., Schwarz, U.D., Schirmeisen, A.: Interfacial friction obtained by lateral manipulation of nanoparticles using atomic force microscopy techniques. J. Appl. Phys. 102, 084306 (2007)

19. Ritter, C., Heyde, M., Stegemann, B., Rademann, K., Schwarz, U.D.: Contact-area dependence of frictional forces: Moving adsorbed antimony nanoparticles. Phys. Rev. B. 71, 085405 (2005)

20. Ogletree, D.F., Carpick, R.W., Salmeron, M.: Calibration of frictional forces in atomic force microscopy. Rev. Sci. Instrum. 67, 3298-3306 (1996)

21. Manini, N., Mistura, G., Paolicelli, G., Tosatti, E., Vanossi, A.: Current trends in the physics of nanoscale friction. Adv. Phys-X 2, 569-590 (2017)

22. de Wijn, A.S.: (In)commensurability, scaling, and multiplicity of friction in nanocrystals and application to gold nanocrystals on graphite. Phys. Rev. B 86, 085429 (2012)

23. Dietzel, D., Brndiar, J., Štich, I., Schirmeisen, A.: Limitations of structural superlubricity: chemical bonds versus contact size. ACS Nano 11, 7642-7647 (2017)

24. Dietzel, D., de Wijn, A.S., Vorholzer, M., Schirmeisen, A.: Friction fluctuations of gold nanoparticles in the superlubric regime. Nanotechnology 29, 155702 (2018)

25. Tartaglino, U., Samoilov, V.N., Persson, B.N.J.: Role of surface roughness in superlubricity. J. Phys. Condens. Matter. 18, 4143 $4160(2006)$

26. He, Q., Joy, D.C., Keffer, D.J.: Impact of oxidation on nanoparticle adhesion to carbon substrates. RSC Adv. 3, 15792-15804 (2013)

Publisher's Note Springer Nature remains neutral with regard to jurisdictional claims in published maps and institutional affiliations.

\section{Authors and Affiliations}

\section{Alper Özoğul ${ }^{1} \cdot$ Benedykt R. Jany ${ }^{2} \cdot$ Franciszek Krok$^{2} \cdot$ Enrico Gnecco $^{1} \cdot$ Mehmet Z. Baykara $^{3}$ (D)}

1 Otto Schott Institute of Materials Research (OSIM), Friedrich Schiller University Jena, 07743 Jena, Germany

2 Marian Smoluchowski Institute of Physics, Jagiellonian University, 30-348 Krakow, Poland
3 Department of Mechanical Engineering, University of California Merced, Merced, CA 95343, USA 